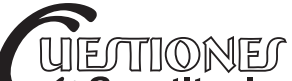 Constitucionales
}

Revista Mexicana de Derecho Constitucional

Núm. 40, enero-junio 2019

\section{¿Hay un derecho al turismo?}

\author{
Is There a Right to Tourism?
}

\section{Antonio MANIATIS*}

RESUMEN: La libertad turística es una versión más amplia de la libertad clásica del movimiento. El derecho al turismo no es nuevo, dado que su existencia es tan antigua como la existencia del turismo. Según ese enfoque, el reconocimiento del turismo es nuevo. El derecho constitucional al turismo se reconoció por primera vez en la versión inicial de la Constitución italiana, que entró en vigor en 1948. Este derecho universal tiene que ver sobre todo con los derechos fundamentales de la segunda generación (particularmente con los derechos sociales, ejemplificados por los derechos al descanso y al ocio), dentro de la cual nació.

Palabras clave: derecho al turismo; garantía institucional; posibilidad jurídica.
ABSTRACT: Tourist freedom is a broader version of the classic freedom of movement. The right to tourism is not new, given that its existence is as old as the existence of tourism. According to this approach, the recognition of tourism is new. The constitutional right to tourism was recognized for the first time in the initial version of the Italian Constitution, which came into force in 1948. This universal right has to do mainly with the fundamental rights of the second generation (particularly with the social rights, exemplified by the rights to rest and to recreation), within which it was born.

Keywords: Right to Tourism; Institutional Guarantee; Juridical Possibility.

* Doctor en Derecho Público; profesor de Derecho Constitucional en la Academia de Bomberos en Grecia; abogado (maniatis@dikaio.gr). 
SUMARIO: I. Aproximación jurídica del turismo. II. ¿Una posibilidad o un derecho de primera generación (derecho civil)? III. ¿Una posibilidad o un derecho de primera generación (derecho político)? IV. ¿Un derecho de segunda generación? V. La evolución del derecho al turismo en la época de la tercera y cuarta generación. VI. La existencia del derecho universal al turismo. VII. Bibliografía.

\section{APROXIMACIÓN JURÍDICA DEL TURISMO}

El turismo ha constituido un fenómeno, en su origen, elitista de unos pocos privilegiados y, después de la Segunda Guerra Mundial, masivo, afectando a la gran mayoría de los países por el mundo entero. ${ }^{1}$ Se trata de una materia multidisciplinaria en conexión estricta con el ocio. Por eso, en el ámbito del derecho, su aproximación científica ha sido lenta y compleja. ${ }^{2}$ El turista es un consumidor aparte, a quien el derecho positivo, con la iniciativa conjunta del legislador y del juez, atribuye siempre más derechos. ${ }^{3}$

En modo similar, otro derecho relativo al ocio ha paralelamente conocido una consagración, aun de orden constitucional. A partir de la década de los setenta, comenzó a reconocerse expresa y gradualmente el derecho al deporte en las Constituciones como emergente derecho económico, social y cultural entonces. ${ }^{4}$ No obstante, ello no condujo a que se construyeran unas doctrinas académicas y jurisprudenciales, que eran necesarias para el desarrollo teórico de la dogmática particular del derecho al deporte, siendo esta última exigida en el propósito de dimensionar correctamente su desarrollo y alcance en su respectiva normativa complementaria.

Existe una amplia gama de leyes que, aunque no son turísticas en esencia, sí tienen relación con la actividad turística. ${ }^{5}$

\footnotetext{
1 Davidson, Rob, Tourism, Singapur, Longman, 1997, pp. 5-7.

2 Jégouzo, Laurence, Le droit du tourisme, Lextenso éditions, 2012, p. 9.

3 Delpech, Xavier, "Les droits du touriste consommateur", Juristourisme, vol. 167, 2014 , p. 29. Por ejemplo, la jurisprudencia comunitaria ha sido favorable a un derecho a indemnización por un retraso en el comienzo de un vuelo por más de tres horas (decisión "Sturgeon").

4 Pachot Zambrana, Karel Luis, "El derecho constitucional al deporte en la doctrina y el derecho comparado", Cuestiones Constitucionales. Revista Mexicana de Derecho Constitucional, núm. 35, julio-diciembre de 2016, p. 147.

5 Jiménez Martínez, Alfonso de Jesús, "La legislación mexicana en torno a la actividad turística", Teoría y Praxis, núm. 4, julio-diciembre de 2007, pp. 99-111.
} 
El turismo se considera como objeto de un derecho relativamente nuevo, mientras que el término "turista" es bastante viejo. ${ }^{6}$ En todo caso, existe la opinión de que la definición, y por lo tanto la existencia, de un "derecho turístico" requeriría, por un lado, la exclusividad de sus normas y, por otro, la aplicación de dichas normas a todas las dimensiones del turismo. ${ }^{7} \mathrm{Mu}-$ chos años antes del comienzo de esta contestación, algunos doctrinarios manejaban la posibilidad de la existencia de un derecho turístico especial (y también transversal, es decir, no exactamente "autónomo"). ${ }^{8}$ La autonomía de una rama separada significaría una gran coherencia interna y una suficiencia metodológica con respecto a sus necesidades de interpretación. ${ }^{9} \mathrm{La}$ opinión de la autonomía del derecho turístico había estado formulada dentro de la doctrina francesa, pero sin seguimiento, en contrapunto con ramas tradicionales como la del derecho público y la del derecho penal. ${ }^{10}$ En todo caso, el derecho turístico ha sido recientemente denominado por algunos, justificadamente o no, "justurismo". ${ }^{11}$ Paralelamente, un nuevo cuerpo de legislación, en este caso sobre la inmigración, ha aparecido como su antípoda conceptual. ${ }^{12}$

El fenómeno de la existencia de muchos nuevos derechos especiales, ejemplificados por los títulos de varios manuales recientemente editados, se considera en el ámbito de la antropología del derecho como prueba de la teoría de la heterogeneidad del derecho, en cuanto al modo de producción de sus normas. La creación de la legislación no es puramente estatal, sino que puede resultar de la sociedad; en este sentido, existe un procedimiento

6 Maniatis, Antonio, “Aspects pénaux et administratifs du droit du tourisme”, $R S C$, 2015, p. 231.

7 Monterrubio, Juan Carlos y Colín, Ricardo, "La inexistencia del derecho turístico. Un análisis conceptual", Estudios y Perspectivas en Turismo, vol. 18, núm. 6, noviembre de 2009, pp. 727-740.

8 Vizcaíno López, Karina, “¿Existe el derecho turístico?”, Ius Revista Jurídica, I-4, 2002, p. 4, disponible en: www.unla.mx/iusunla4/.

9 Lachièze, Christophe, Droit du tourisme, París, LexisNexis, 2014, p. 17 y n. 89. Es obvio que pocas ramas, dotadas con una gran tradición, podrían considerarse como autónomas, como es el caso del derecho mercantil.

10 Servoin, François, Institutions touristiques et droit du tourisme, Masson, 1981, p. 12.

11 Armas Morales, Carlos, "El justurismo", Gestión en el Tercer Milenio, año 7, núm. 13, 2004, pp. 39-43.

12 Papakonstantinou, Apóstolos, "Inmigrantes y derechos fundamentales", Unión por la defensa de los derechos sociales, 2007 (en griego), disponible en: http://www.epkodi.gr/ site/index.php/------articlestable-83/52----. 
oficial, relativo a los órganos públicos competentes, y otro informal e interno de las comunidades sociales interesadas. ${ }^{13}$

El presente análisis se focaliza en la cuestión respecto a si hay un derecho, de tipo jurídico clásico, o una mera posibilidad humana, en materia de la actividad turística. En primer lugar, se trata de una investigación sobre la naturaleza eventual del turismo como objeto de derecho civil, de primera generación. Enseguida, esta investigación se cumple con la temática del mismo derecho como derecho político, dentro de la misma generación. De igual forma, sería oportuno analizar si se trata también de un derecho de segunda generación. Después de esta reseña histórica, es apropiado examinar la cuestión de esa garantía eventual durante la tercera y la cuarta generación. Finalmente, sigue una conclusión sobre la naturaleza global del derecho eventual al turismo.

\section{II. ¿UNA POSIBILIDAD O UN DERECHO DE PRIMERA GENERACIÓN (DERECHO CIVIL)?}

No podría darse por sentado que hay un derecho al turismo simplemente teniendo en cuenta el hecho de que la actividad turística no está prohibida y que en muchos ordenamientos jurídicos nacionales está regulada por normas específicas de la legislación.

Con base en esos datos, es posible reconocer una posibilidad jurídica de desarrollar las actividades turísticas. Por ejemplo, el derecho comunitario europeo hacía uso del término "derecho" para indicar el derecho de petición al Parlamento Europeo. Sin embargo, la creación de una posibilidad similar a la denuncia de casos de mala administración comunitaria al defensor del pueblo europeo, órgano auxiliar y también autónomo del Parlamento, no estuvo combinada con la misma terminología. El artículo $8 \mathrm{D}$ del Tratado de la Comunidad Europea mencionaba que cada ciudadano puede dirigirse al mediador. Entonces, pese al hecho de que, en principio, la queja al defensor tiene que ver con temas menos políticos que los temas tratados por la petición sometida al Parlamento, es ella misma objeto de los derechos atribuidos al ciudadano europeo por primera vez con el Tratado de la Unión Europea. No obstante, la doctrina estima que la denominación "posibilidad", en lugar del término "derecho", no produce

\footnotetext{
13 Rouland, Norbert, L'anthropologie juridique, PUF, 1995, pp. 68-71.
} 
resultados jurídicos diferentes en la práctica. En todo caso, no está excluida la eventualidad de convertir oficialmente una "posibilidad" en un "derecho". Por ejemplo, a nivel de la Unión Europea o estatal, la petición al defensor del pueblo y aquella al Parlamento podrían constituir objeto de un derecho fundamental único, al servicio de los interesados. ${ }^{14}$

En esencia, el derecho al turismo está históricamente basado en un derecho civil originado de la libertad del desplazamiento. El término eleftheria, es decir, la palabra griega sinónima del vocablo "libertad", significa, desde un punto de vista etimológico, "la venida" y, en consecuencia, la facultad corporal de moverse de un lado a otro. En conclusión, la libertad turística es, en su forma y esencia elemental, una versión más amplia de la libertad del movimiento. En términos jurídicos, se trata de una forma del derecho civil (libertad civil individual) del desplazamiento. Esta constatación está sugerida a nivel de la doctrina que sostiene la opinión de que el derecho al turismo no es nuevo, dado que su existencia es tan antigua como la existencia del turismo. Según ese enfoque, el reconocimiento del turismo es nuevo. ${ }^{15}$ Por eso, es posible hacerle entrar al "panteón" de los nuevos derechos como aquello de las nuevas tecnologías, del medio ambiente, del consumo o, incluso, de la energía. ${ }^{16}$

Interpretando la teoría citada sobre el carácter diacrónico del derecho turístico, el derecho al turismo ha sido tradicionalmente una "posibilidad" en un sentido amplio, conocida desde los tiempos de la antigüedad. En un principio, se trataba simplemente de una libertad anónima, es decir, una posibilidad no prohibida de manera legal. Después, la libertad anónima fue promocionada a una "posibilidad" jurídica de los individuos, explícitamente reconocida. En este procedimiento histórico, la estación más reciente tiene que ver con la evolución de la transformación de la "posibilidad" en un "derecho". Hoy día, no se trata solamente de un "derecho", sino también de un derecho fundamental, de naturaleza civil, dentro de la lista de varios derechos nuevos, con un reconocimiento constitucional explícito o no.

14 Maniatis, Antonio, Le recours parlementaire dans l'Union Européenne, Éditions Ant. N. Sakkoulas, 2000, p. 317.

15 Jégouzo, Laurence, "Le droit du tourisme: un droit en gestation?", Mélanges Yves Jégouzo, Dalloz, 2009. Es obvio que el turismo constituye una libertad, pero la cuestión es cuándo ha adquirido la forma de derecho consagrado en el derecho positivo.

16 Jégouzo, Laurence, "Le droit du tourisme est-il un droit nouveau?", Juristourisme, núm. 145, 2012, p. 22. 


\section{III. ¿UNA POSIBILIDAD O UN DERECHO DE PRIMERA GENERACIÓN (DERECHO POLÍTICO)?}

La posibilidad del turismo no sólo ha sido una libertad civil, sino también política, ejemplificando así la teoría del carácter complementario de los estatutos de los derechos fundamentales. En realidad, ese derecho tiene una dimensión política, sobre todo en el caso de las visitas en el extranjero. Por ejemplo, el turismo internacional ha sido motor de cambio y relativo factor de legitimación del régimen franquista, que estuvo sancionado por las Naciones Unidas hasta $1950 .{ }^{17}$ Sin embargo, ha operado paulatinamente la función aun de un factor de desestabilización informal de un régimen político muy diferente del modelo constitucional de los países de origen de los visitadores de España. El general Franco falleció el 20 de noviembre de 1975. El sistema que había creado difícilmente podía sobrevivirle, pues se apoyaba en la personalidad y en la autoridad de aquél y, además, porque sectores más o menos amplios de la sociedad española se sentían marginados del mismo. ${ }^{18}$

En todo caso, regímenes autoritarios como la dictadura de Juan Metaxas, establecida en 1936 en Grecia, tienen la tendencia de intentar controlar la cultura de los individuos y de su manifestación pública, como en la materia de la comunicación masiva y de los intercambios turísticos. Por ello, en la Ley coercitiva 45/1936, "De creación de una Secretaría de Prensa y Turismo", se usó la expresión "cuestiones relativas a la iluminación de la opinión pública", por lo que se refiere a esa materia legislativa. Por primera vez en la historia del Estado griego se fundó dentro del gobierno una unidad, de orden casi ministerial, competente para esas áreas de interés común. La iniciativa de una dictadura que admiraba al régimen fascista de Italia sugiere el carácter cultural de ambos fenómenos; principalmente, demuestra la fisionomía política de los derechos humanos al turismo y a la información de la sociedad con la prensa. El turismo no constituye una actividad apolítica, indiferente por el poder político, según la experiencia originada de la tendencia de su manipulación centralizada por la gobernación autoritaria. En consecuencia, el principio tradicional de la libertad de prensa frente al Estado y el derecho al turismo - no tan viejo — tienen una naturaleza jurídica común: civil, sobre todo, y política.

17 Rodríguez Rivero, Manuel, "Los turistas de Franco”, El País, 2009.

18 Villarroya, Joaquín Tomás, Breve historia del constitucionalismo español, Madrid, Centro de Estudios Constitucionales, 1990, p. 160. 
En ocasiones, la fisionomía política de estos fenómenos, los cuales pueden jugar un papel importante en la historia, está acentuada por la historia misma. Éste es el caso del ordenamiento jurídico helénico, en el cual la comunicación masiva fue objeto explícito de un ministerio, como fue el turismo (ya convertido en un fenómeno masivo). En el periodo 19941996, el Ministerio del Turismo coexistió con el Ministerio de Prensa y de Medios de Comunicación Masiva. Dos generaciones después del hecho arquetípico de la creación de una Secretaría de Prensa y Turismo, los dos objetos "se han encontrado" en el mismo esquema de gobierno, correspondiendo a ministerios especializados y separados.

\section{IV. ¿UN DERECHO DE SEGUNDA GENERACIÓN?}

En materia del derecho al turismo, los legisladores, en principio, no están habituados al uso explícito del término "derecho al turismo"; sin embargo, esta abstención no debería interpretarse como una negación a la existencia de un derecho al turismo. La abstención podría atribuirse a varias razones, por ejemplo, la edad relativamente pequeña de ese derecho, en distinción a otros derechos clásicos, como el derecho de la petición parlamentaria, y la particularidad en la consagración de los derechos fundamentales de "segunda generación". Se trata de los derechos sociales, los cuales, en principio, estuvieron por primera vez institucionalizados después de la Primera y, sobre todo, la Segunda Guerra Mundial. Dado que esta nueva serie de derechos, los cuales están relacionados a la calidad de vida de la gente, implica un cargo institucional y, principalmente, financiero importante del sector público, el legislador ha preferido una formulación conservativa para sugerir la existencia de tales derechos. Por ejemplo, hace uso de términos como "obligación" o "preocupación" del Estado por bienes legales como el medio ambiente y el turismo. Mediante la revisión de la Constitución griega de 2001, se introdujo explícitamente el principio del Estado social de derecho, que rige la materia de los derechos constitucionales, como el derecho al turismo, hasta hoy no mencionado en esta Constitución.

Por lo que se refiere a la segunda generación de derechos, no se trata únicamente de derechos sociales, sino también de derechos a la economía (trabajo, sindicalismo, huelga, etcétera) y a la cultura (arte, atletismo, entre 
otros). ${ }^{19}$ Por ejemplo, el deporte no fue objeto de atención expresa por alguna Constitución hasta que finalizó la Segunda Guerra Mundial, cuando con la definitiva consolidación de los constitucionalismos social y socialista comenzó a manifestarse un marcado interés por el deporte, encuadrándose inicialmente entre los objetivos principales de atención pública. ${ }^{20}$ Los diversos aspectos de la cultura, desde los monumentos antiguos a los juegos deportivos, concentraron el interés de los turistas, de manera particular en el cuadro del movimiento desarrollado en esos años: el turismo masivo. Si la cultura ha sido, en ese sentido, una atracción fuerte desde el punto de vista turístico, los nuevos derechos constitucionales relativos a las actividades económicas, dotados con una sensibilidad social, han creado las condiciones socioeconómicas de los desplazamientos turísticos sistematizados. Así, una ley de 1936 había previsto por primera vez en el ordenamiento francés el derecho a vacaciones pagadas. Los derechos al descanso y al ocio constituyen una conquista jurídica de la era de la segunda generación.

Cabe señalar que el turismo mismo constituye una garantía constitucional (derecho y, sobre todo, garantía institucional) de esa era, dado que la Constitución italiana, la cual entró en vigor el 1o. de enero de 1948, fue la primera en hablar del "turismo" y de la "industria hotelera" (como materia legislativa para la cual las regiones con régimen ordinario serían competentes). ${ }^{21}$ Por lo que se refiere a la expresión "garantía institucional" introducida en aquella época, se trata de un término que no está explícitamente consagrado en las Constituciones. Asimismo, éste constituye una forma de protección; por ejemplo, tiene la forma de libertad-oferta a personas de cierta propiedad, no para proteger a las personas como individuos, sino para asegurar la existencia independiente y la misión exenta de obstáculos de la institución que esas personas representan. Se trata de mecanismos de protección de ciertos aspectos del poder estatal, ejemplificados por el principio de independencia de los jueces o de la sociedad. Es importante señalar que muchas garantías del segundo grupo tienen un impacto constitutivo en el sistema político, como la libertad de la prensa (llamada "cuarto poder") y el movimiento turístico, sobre todo el turismo masivo, según el precedente análisis del estatuto político del derecho fundamental al turismo.

19 Del Giudice, Federico, Compendio di diritto costituzionale, 20a. ed., Edizioni Giuridiche Simone, 2016, p. 102.

20 Pachot Zambrana, Karel Luis, op. cit., p. 123.

21 Maniatis, Antonio, "The Right to Pursuit of Happiness and Italian Tourism Law", Tourism Development Journal, vol. 15, núm. 1, 2017, p. 54. 


\section{LA EVOLUCIÓN DEL DERECHO AL TURISMO EN LA ÉPOCA \\ DE LA TERCERA Y CUARTA GENERACIÓN}

Algunos derechos tradicionalmente considerados por la doctrina como sociales están consagrados con una formulación mixta, es decir, como derechos y deberes de sus sujetos, por ejemplo, el derecho de proteger y mantener el medio ambiente o el derecho al trabajo en la Constitución de la República Bolivariana de Venezuela, adoptada en 1999.

En todo caso, existió una entera y nueva generación de derechos fundamentales entre 1972 (año de surgimiento del derecho al medio ambiente) y 1992 (año de reconocimiento del derecho a contener el cambio climático). La tercera generación, que proviene del derecho internacional, tiene que ver con la fraternidad, contemplada dentro del tríptico de la Revolución francesa: "Libertad, Igualdad, Fraternidad". A su vez, se incluyen varios derechos de solidaridad entre los hombres o entre los pueblos, como los derechos al disfrute y a la protección del medio ambiente, al desarrollo, a la paz, al patrimonio (natural y cultural) común de la humanidad, etcétera. ${ }^{22}$ Como ya se señaló, estos derechos tienen una naturaleza más o menos mixta, es decir, se consideran como "deberes" o "responsabilidad" de las personas. Por ejemplo, el derecho a la paz también implica el deber de los individuos o de los pueblos de actuar de modo pacífico (a través de la educación de las nuevas generaciones, de la práctica de no violencia, etcétera). ${ }^{23}$ Asimismo, los derechos de solidaridad son derechos colectivos, en el sentido de que pertenecen a colectividades, como son los pueblos o los grupos sociales, en combinación con las futuras generaciones de la humanidad, por ejemplo, por lo que se refiere al principio moderno de la sostenibilidad del medio ambiente. ${ }^{24}$ La materia turística pertenece a esa lista de "responsabilidad" social de los ciudadanos, porque no tiene que ver con la mera gestión individual del tiempo libre, sino con el uso de los bienes ambientales con base en el principio (derecho de tercera generación) del desarrollo sustentable.

El turismo tiene una posición constitucional implícita, ejemplificada por la Constitución griega, o relativamente limitada en la gran mayoría de las

22 Chrysogonos, Constantinos, Derechos civiles y sociales, 3a. ed., Nomiki Vivliothiki, 2006, p. 43 (en griego).

23 De Stefani, Paolo, "Diritti umani di terza generazione", Aggiornamenti Sociali, núm. 1, 2009, p. 21.

24 Ibidem, p. 19. 
Constituciones. Por ejemplo, la Constitución Española, que entró en vigor en 1978, en su artículo 148, prevé que las comunidades autónomas podrán asumir competencias en ciertas materias, como "la promoción y ordenación del turismo en su ámbito territorial" 25 y la "promoción del deporte y de la adecuada utilización del ocio". De acuerdo con la decisión tomada por cada uno de los diecisiete legisladores en la materia turística, las comunidades han asumido competencias exclusivas en virtud de lo dispuesto en este artículo. Sin embargo, el turismo debería considerarse como una cuestión, principalmente, del ámbito de la competencia del Estado, es decir, una actividad de interés nacional (central). El legislador estatal tiene competencias normativas exclusivas en materia de legislación civil y mercantil según, el artículo 149; además, sobre la base de este artículo, ha podido regular mediante ley el régimen jurídico de los viajes combinados y de los derechos de aprovechamiento por turno de bienes inmuebles de uso turístico.

Durante la época corriente de la cuarta generación de consagración de los derechos fundamentales, ejemplificada por los derechos en materia de los tratamientos genéticos y la bioética, de las nuevas tecnologías de comunicación y del mundo de los animales, ${ }^{26}$ el derecho constitucional al turismo desapareció en su "patria". La introducción explícita del turismo en la Constitución italiana ha sido eliminada por la revisión de 2001, pero sin implicar un cambio de competencias entre el Estado y las regiones. El turismo sigue siendo no sólo un derecho fundamental, sino también, al menos en algunos ordenamientos constitucionales dotados con una mención explícita del turismo, una garantía institucional. La garantía de la institución turística resulta de principios constitucionales explícitos, como la sustentabilidad del medio ambiente natural y cultural, particularmente del patrimonio arqueológico, ${ }^{27} \mathrm{y}$ el Estado social del derecho. Ambos principios han sido explícitamente consagrados en la Constitución griega. ${ }^{28}$ No obstante, es preferible adoptar una reglamentación especializada, como aquella de la Constitución de la República Bolivariana de Venezuela, de 1999. Según el artículo 310:

25 Imitando a la Constitución italiana.

26 Del Giudice, Federico, Compendio di diritto costituzionale, 20a. ed., Edizioni Giuridiche Simone, 2016, p. 102.

27 Drouet, Marc, "Le patrimoine archéologique pour tous", Juristourisme, núm. 165, 2014, pp. 37-39.

28 Maniatis, Antonio, "PPP and the Constitutional Right to the Environment", Constitutionalism.gr, 2015, p. 2. 
El turismo es una actividad económica de interés nacional, prioritario para el país en su estrategia de diversificación y desarrollo sustentable. Dentro de las fundamentaciones del régimen socioeconómico previsto en esta Constitución, el Estado dictará las medidas que garanticen su desarrollo. El Estado velará por la creación y fortalecimiento del sector turístico nacional'.

Es notable que el derecho al turismo se ha promocionado mucho a través de su consagración en el Código Ético Mundial para el Turismo. Aprobado en 1999 por la Asamblea General de la Organización Mundial del Turismo, ese texto no es jurídicamente vinculante, sino que incorpora un mecanismo de aplicación voluntaria. De acuerdo con el párrafo 2 del artículo 7o.:

El derecho al turismo para todos debe entenderse como consecuencia del derecho al descanso y al ocio, y en particular a la limitación razonable de la duración del trabajo y a las vacaciones pagadas periódicas, que se garantiza en el artículo 24 de la Declaración Universal de los Derechos Humanos y en el artículo 7.d del Pacto Internacional de Derechos Económicos, Sociales y Culturales.

Esta aproximación del derecho universal al turismo es útil para el descubrimiento del parentesco más importante de este derecho con otros grupos de derechos fundamentales. El derecho al turismo, como garantía constitucional explícitamente consagrada en el derecho positivo, tiene que ver, sobre todo, con los derechos de la segunda generación (particularmente con los derechos sociales), dentro de la cual nació por primera vez.

\section{LA EXISTENCIA DEL DERECHO UNIVERSAL AL TURISMO}

El derecho humano al turismo no sólo existe, sino que además implica una deontología turística, ejemplificada por el deber (de las agencias de viajes) de informar y de desaconsejar a los turistas la visita de destinos peligrosos; ${ }^{29} \sin$ embargo, no está consagrado suficientemente, por lo que se refiere a su reconocimiento explícito en la gran mayoría de las Cons-

29 Vainopoulos, Richard y Mercier, Sandrine, Le tourisme, París, Le Cavalier Bleu Éditions, 2009, p. 71. 
tituciones formales, pese a la contribución de muchos textos a la lista de derechos constitucionales, particularmente de los nuevos. ${ }^{30}$ Además, se usa en textos constitucionales, como el español, una variedad terminológica que hace difícil una sistematización de los derechos, por lo general. ${ }^{31} \mathrm{En}$ consecuencia, sería oportuno fortalecer las garantías constitucionales de varios tipos en esa materia, así como en lo relativo al principio del Estado social de derecho. ${ }^{32}$

El procedimiento de la consagración del derecho al turismo pone de relieve:

- La transformación de las "posibilidades" de los privados en derechos civiles y derechos políticos clásicos (por ejemplo, el derecho de formar y expresar opinión por escrito, oralmente o mediante la prensa).

- La transformación de esas "posibilidades", en ocasiones, en "garantías institucionales", habitualmente de carácter político (como es el caso de la libertad de la prensa).

- La proyección de los derechos sociales en la sociedad por medio de la creación de la tercera generación — solidaridad de un grupo social (o de un pueblo) a otro-, mientras que comentarios similares se aplican a los derechos de la cuarta generación, como es el caso del derecho a contener el cambio climático.

En este orden de ideas, la "posibilidad" de hacer turismo se ha promocionado en un derecho universal perteneciente a la segunda generación de los derechos fundamentales, sobre todo asociado a los derechos sociales, particularmente al descanso y al ocio.

La temática del derecho al turismo pone de relieve la cuestión del derecho a la hospitalidad, el cual tiene un reconocimiento aún marginal.

30 Häberle, Peter, La teoría de las etapas de evolución de los textos, Atenas-Komotini, Ediciones Ant. N. Sakkoula, 1992, p. 44 (traducción en griego).

31 Rolla, Giancarlo, "Il sistema costituzionale dei diritti fondamentali. L'esperienza spagnola”, Rivista Trimestrale di Diritto Pubblico, núm. 1, 1990, p. 145.

32 La Torre, Michele, "Le garanzie costituzionali nella nuova Costituzione", en Camera dei Deputati, Il centenario del Parlamento: 8 maggio 1848-8 maggio 1948, Roma, Camera dei Deputati, 1948, p. 442; Giner, Carlos, El defensor del pueblo en la teoría y en la práctica, Madrid, Popular, 1986, pp. 9 y 10. 


\section{BIBLIOGRAFÍA}

Armas Morales, Carlos, "El justurismo”, Gestión en el Tercer Milenio, año 7, núm. 13, 2004.

Chrysogonos, Constantinos, Derechos civiles y sociales, 3a. ed., Nomiki Vivliothiki, 2006.

DAVIDSON, Rob, Tourism, Singapur, Longman, 1997.

De Stefani, Paolo, "Diritti umani di terza generazione", Aggiornamenti Sociali, núm. 1, 2009.

DELPECH, Xavier, "Les droits du touriste consommateur", Juristourisme, vol. 167, 2014.

Del GiUdice, Federico, Compendio di diritto costituzionale, 20a. ed., Edizioni Giuridiche Simone, 2016.

DEL VeCCHIO, Giorgio, Studi sullo stato, Milán, Giuffrè Editore, 1958.

DrouET, Marc, "Le patrimoine archéologique pour tous", Juristourisme, núm. 165, 2014.

GINER, Carlos, El defensor del pueblo en la teoría y en la práctica, Madrid, Popular, 1986.

HÄBERLE, Peter, La teoría de las etapas de evolución de los textos, AtenasKomotini, Ediciones Ant. N. Sakkoula, 1992.

JÉGOUZO, Laurence, Le droit du tourisme, Lextenso éditions, 2012.

JÉGOUZO, Laurence, "Le droit du tourisme: un droit en gestation?", Mélanges Yves Jégouzo, Dalloz, 2009.

JÉGOUZO, Laurence, “Le droit du tourisme est-il un droit nouveau?", Juristourisme, núm. 145, 2012.

JiMÉNEZ MARTínEZ, Alfonso de Jesús, "La legislación mexicana en torno a la actividad turística”, Teoría y Praxis, núm. 4, julio-diciembre de 2007.

LACHIÈZE, Christophe, Droit du tourisme, París, LexisNexis, 2014.

LA TORRE, Michele, "Le garanzie costituzionali nella nuova Costituzione", en Camera dei Deputati, Il centenario del Parlamento: 8 maggio 1848-8 maggio 1948, Roma, Camera dei Deputati, 1948.

MANIATIS, Antonio, “Aspects pénaux et administratifs du droit du tourisme", RSC, 2015.

MANIATIS, Antonio, Le recours parlementaire dans l'Union Européenne, Éditions Ant. N. Sakkoulas, 2000. 
MANiATIS, Antonio, "PPP and the Constitutional Right to the Environment", Constitutionalism.gr, 2015.

MAniatis, Antonio, "The Right to Pursuit of Happiness and Italian Tourism Law”, Tourism Development Journal, vol. 15, núm. 1, 2017.

Monterrubio, Juan Carlos y Colín, Ricardo, "La inexistencia del derecho turístico. Un análisis conceptual”, Estudios y Perspectivas en Turismo, vol. 18, núm. 6, noviembre de 2009.

PAPAKONSTANTINOU, Apóstolos, "Inmigrantes y derechos fundamentales", Unión por la defensa de los derechos sociales, 2007.

PACHOt ZAMBRANA, Karel Luis, "El derecho constitucional al deporte en la doctrina y el derecho comparado", Cuestiones Constitucionales. Revista Mexicana de Derecho Constitucional, núm. 35, julio-diciembre de 2016.

Rodríguez Rivero, Manuel, “Los turistas de Franco”, El País, 2009.

Rolla, Giancarlo, "Il sistema costituzionale dei diritti fondamentali. L'esperienza spagnola", Rivista Trimestrale di Diritto Pubblico, núm. 1, 1990.

RoUlAND, Norbert, L'anthropologie juridique, PUF, 1995.

SERVOIN, François, Institutions touristiques et droit du tourisme, Masson, 1981.

VAinopoulos, Richard y Mercier, Sandrine, Le tourisme, París, Le Cavalier Bleu Éditions, 2009.

VILlarroya, Joaquín Tomás, Breve historia del constitucionalismo español, Madrid, Centro de Estudios Constitucionales, 1990.

VIZCAÍNO LÓPEZ, Karina, “¿Existe el derecho turístico?”, Ius Revista Jurídica, I-4, 2002.

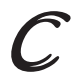

Fecha de recepción: 15 de octubre de 2016.

Fecha de aceptación: 24 de agosto de 2018. 